\title{
Interaction-induced delocalization of quasiparticle pairs in the Anderson insulator
}

\author{
Felix von Oppen* and Tilo Wettig \\ Max-Planck-Institut für Kernphysik, 69117 Heidelberg, Germany
}

(November 7, 1995)

\begin{abstract}
It has recently been established that a short-range interaction can strongly delocalize a pair of particles moving in a disordered potential. We investigate whether an analogous effect exists also for pairs of quasiparticles in the Anderson insulator by employing an approximate numerical evaluation of the two-particle Green function of the many-body system. At the Fermi energy, the quasiparticle pair is localized on the scale of the single-particle localization length. The delocalization effect is recovered when the excitation energy of the pair is comparable to the bandwidth.
\end{abstract}

PACS numbers: 72.15.R, 71.30.

The localization of particles by a random potential has been studied extensively over the past decades [1]. While wavefunctions are exponentially localized in one and two dimensions even for arbitrarily weak disorder, there exists a transition between extended and localized states in three dimensions. A non-interacting degenerate Fermi gas in a disordered potential is conducting when the Fermi energy lies in the region of extended states (metallic phase) while it is insulating when the Fermi energy lies in the region of localized states (Anderson insulator). The situation becomes much more complex and controversial once interactions between the particles are included. Interactions may lead to a metal-insulator transition (Mott transition) even in the absence of disorder; they also result in rich magnetic behavior [2]. Interest in the interplay of disorder and interactions was recently renewed in part by experiments measuring persistent currents in mesoscopic normal-metal rings whose amplitude could not be explained by theories for noninteracting electrons [3].

An original approach to studying localization in the presence of interactions was recently taken by Shepelyansky [4] and previously by Dorokhov [5]. They considered the much simpler problem of two interacting particles in a random potential and predicted that the interaction can lead to a significant delocalization of the pair. Shepelyansky [4] and Imry [6] speculated that an analogous effect could exist for pairs of quasiparticles at finite particle density which may have significant consequences for the thermodynamic and transport properties of the Anderson insulator. It is the purpose of the present paper to study the propagation of quasiparticle pairs in the Anderson insulator in the presence of a short-range interaction within a numerically tractable approximation to the twoparticle Green function. Our approximation is motivated by both physical and analytical considerations.

We briefly recall some of the pertinent results for the two-particle problem. Shepelyansky [4] studied two particles in a random potential interacting by a shortrange interaction. Whenever the two particles are local- ized far apart compared to the single-particle localization length $\xi_{1}$, the effect of the interaction is only exponentially small. By contrast, the two particles can propagate as a pair over a distance $\xi_{2}$ which can be much larger than $\xi_{1}$ when they are localized within about one single-particle localization length of each other. Specifically, Shepelyansky predicted by an approximate mapping to a banded-random-matrix model which he studied numerically that the two-particle localization length $\xi_{2}$ satisfies $\xi_{2} / \xi_{1} \simeq\left(\xi_{1} / 32\right)(u / t)^{2}$, where $u$ denotes the disorder strength and $t$ the bandwidth (hopping matrix element). The effect is essentially independent of the sign of the interaction and the statistics of the particles. Subsequently, the existence of this effect was confirmed using both a Thouless-type scaling argument [6] and numerical calculations [7, 8]. Numerical work \&8] computing $\xi_{2}$ directly from the Green function of the twoparticle problem showed that in strictly one dimension the two-particle localization length at the center of the band satisfies a scaling relation $\xi_{2} / \xi_{1}=f\left(u \xi_{1} / t\right)$ with $f(x) \simeq 1 / 2+C|x|$ which, while qualitatively confirming the effect, is inconsistent with the original prediction mentioned above ( $C$ is a numerical constant).

The most important additional feature of the degenerate Fermi system is the Pauli principle. Two particles propagating in a disordered potential sufficiently far from the band edges have ample phase space for scattering. On the other hand, phase space for scattering is severely restricted at finite density since all states below the Fermi energy are effectively blocked. One may expect that this leads to a suppression of the delocalization at low pair excitation energy. With increasing excitation energy, the phase-space restriction becomes less severe and the delocalization effect should be recovered. We study the influence of the Pauli principle on the propagation of quasiparticle pairs within an approximation analogous to the Cooper problem of superconductivity: We consider two particles above the Fermi energy which interact with each other, but not with the electrons in the Fermi sea except via the exclusion principle. The Fermi 


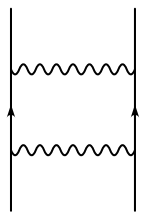

(a)

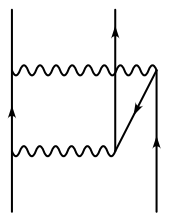

(b)

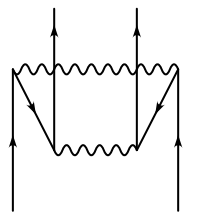

(c)
FIG. 1. Examples of elementary second-order diagrams in the particle-particle channel with time running upwards. While diagram (a) contributes to leading order in the low-density limit, diagrams (b) and (c) are suppressed since they involve particle-hole-pair creation and annihilation.

sea is defined by filling up single-particle eigenstates of the disorder potential below the Fermi energy $E_{F}$. In order to avoid the superconducting instability, we consider only repulsive interactions.

One can motivate this approximation also by a quite different line of thought. It is plausible that the twoparticle effect is most likely to survive at low particle density. When considering a short-range interaction in three dimensions, only ladder diagrams involving no intermediate hole excitations contribute to leading order in the low-density limit 9]. Hence, the Cooper problem is in fact equivalent to a low-density approximation. This can be understood physically by considering some second-order diagrams as shown in Fig. 1. While the ladder diagram in Fig. 1(a) involves only particle excitations at the intermediate stage, the diagrams 1(b) and 1(c) involve intermediate hole excitations. The latter diagrams are suppressed at low density because they involve particle-hole-pair creation. For these reasons, we will only study particle-particle excitations in this paper. We expect that our results apply qualitatively also to hole-hole and particle-hole excitations.

We now proceed to work out the Cooper approximation within the diagrammatic approach. We consider spinless fermions on a one-dimensional lattice with repulsive nearest-neighbor interaction of strength $u$ and subject to a random potential,

$$
\begin{aligned}
\hat{H}= & t \sum_{n}\left[\hat{a}_{n}^{\dagger} \hat{a}_{n+1}+\hat{a}_{n+1}^{\dagger} \hat{a}_{n}\right]+\sum_{n} v_{n} \hat{a}_{n}^{\dagger} \hat{a}_{n} \\
& +u \sum_{n} \hat{a}_{n}^{\dagger} \hat{a}_{n+1}^{\dagger} \hat{a}_{n+1} \hat{a}_{n} .
\end{aligned}
$$

Here, $\hat{a}_{n}$ denotes the fermionic annihilation operator of a particle at site $n$, and $v_{n} \in[-W / 2, W / 2]$ denotes the random on-site energies. In the following, energies will be measured in units of the hopping matrix element $t$ and lengths in units of the lattice spacing $a$. As motivated above, we focus on the retarded two-particle Green function in the particle-particle channel. Analogous to our approach [8] to the two-particle problem, we consider only processes where the quasiparticles are created on neighboring sites $(m, m+1)$ at time $\tau=0$ and destroyed at time $\tau$ on a different set of neighboring sites $(n, n+1)$. The corresponding amplitude is given by

$$
\begin{aligned}
F^{\mathrm{pp}}(n, m ; \tau) & =-i \theta(\tau)\left\langle 0\left|\hat{a}_{n}(\tau) \hat{a}_{n+1}(\tau) \hat{a}_{m+1}^{\dagger}(0) \hat{a}_{m}^{\dagger}(0)\right| 0\right\rangle \\
& =\int_{-\infty}^{\infty} \frac{d E}{2 \pi} F^{\mathrm{pp}}(n, m ; E) \exp (-i E \tau / \hbar) .
\end{aligned}
$$

The localization length $\xi_{q}$ for coherent propagation of the quasiparticle pair can be defined by the exponential decrease of these matrix elements with distance,

$$
\frac{1}{\xi_{q}(E)}=-\lim _{|n-m| \rightarrow \infty} \frac{1}{|n-m|}\left\langle\ln \left|F^{\mathrm{pp}}(n, m ; E)\right|\right\rangle,
$$

where $\langle\ldots\rangle$ denotes a disorder average. When considering the nearest-neighbor interaction in (11), a closed equation can be derived for the matrix elements $F^{\mathrm{pp}}(n, m ; E)$ within the ladder approximation. It is this enormous simplification which makes our numerical calculations feasible.

Computing $F^{\mathrm{pp}}(n, m ; E)$ for non-interacting fermions, one readily obtains

$$
F_{0}^{\mathrm{pp}}(n, m ; E)=\sum_{\epsilon_{i}>\epsilon_{j}>E_{F}} \frac{B_{i, j}^{*}(n) B_{i, j}(m)}{E-\epsilon_{i}-\epsilon_{j}+i \eta} .
$$

Here, $\epsilon_{i}$ denotes the single-particle energy of the eigenstate $\phi_{i}(n)$ of the disorder potential, $\eta$ is a positive infinitesimal, and $B_{i, j}(n)=\phi_{i}(n) \phi_{j}(n+1)-\phi_{i}(n+1) \phi_{j}(n)$. Summing the ladder diagrams and considering only the contributions of intermediate particle-particle states, one obtains the two-particle Green function in the Cooper approximation,

$$
F^{\mathrm{pp}}(E)=\frac{F_{0}^{\mathrm{pp}}(E)}{u} \frac{1}{1 / u-F_{0}^{\mathrm{pp}}(E)},
$$

where $F^{\mathrm{pp}}(E)$ is viewed as a matrix in site space. It is instructive to compare these equations to those for the two-particle problem [B]. The difference between the two cases is that the sum in (14) is only over states above the Fermi energy while the corresponding equation for the two-particle case involved an unrestricted summation over all states. It is possible to extend our approach to the full ladder approximation. Then, Eq. (4) would also contain a summation over hole-hole excitations. However, we decided to use the Cooper approximation because it can be motivated systematically in the low-density limit. We do not expect that our results change qualitatively when hole-hole excitations are included.

The first factor on the right-hand side of (5) decreases on the scale of the single-particle localization length. Hence, we focus on the second factor in the following from which any long-range behavior must arise. For numerical purposes, it is instructive to interpret this term 
as the Green function for the "Hamiltonian" $F_{0}^{\mathrm{pp}}(E)$ at "energy" $1 / u$. Since $F_{0}^{\mathrm{pp}}(E)$ is a banded matrix whose bandwidth is of the order of the single-particle localization length, this allows us to compute the localization length $\xi_{q}$ by employing the efficient recursive Greenfunction technique for banded Hamiltonian matrices [10].

For our numerical calculations we chose a Fermi energy $E_{F}=-1$, corresponding roughly to quarter filling. We have studied the localization length of quasiparticle pairs for nine values of the pair energy $E$ ranging from $E=-2$ to $E=0$ in steps of $\Delta E=0.25$. This corresponds to excitation energies $\epsilon=E-2 E_{F}$ of the quasiparticle pair between $\epsilon=0$ and $\epsilon=2$. For each value of the excitation energy we used three values of disorder $W=1.5,2$, and 3 , corresponding to single-particle localization lengths at the band center $\xi_{1}=46.7,26.2$, and 11.7 , respectively, and five values of the interaction $u=0.1,0.3,0.5,0.75$ and 1.0. For comparison, we have also computed $\xi_{2}$ of the two-fermion problem for the same set of parameters. The computations were done for lattices with 500 sites, and we averaged over 50 realizations of the disorder. We briefly comment on our choice of $E_{F}$. As argued above, our approximation can be motivated by the low-density limit. In view of this, our choice of $E_{F}$ may seem quite large. We chose this somewhat larger value of $E_{F}$ since the two-particle effect becomes increasingly weaker as one moves away from the center of the band, mostly because the single-particle localization length decreases. Hence, for smaller values of $E_{F}$, it would be difficult to identify a suppression of the delocalization effect relative to the two-particle effect.

In Fig. 2 we exhibit our results for the two pair energies $E=-2$ and $E=0$ corresponding to excitation energies $\epsilon=0$ and $\epsilon=2$, respectively. We plot $\xi_{q}(E) / \xi_{1}(E / 2)$ as a function of $u \xi_{1}(E / 2) / t$ analogous to the scaling plot for the two-particle problem. While we do not find the same scaling any more in the many-particle problem, this still allows us to exhibit data for all values of the interaction and the disorder strength in a single plot. Clearly, the delocalization effect disappears almost completely for zero excitation energy.t. On the other hand, the delocalization of the quasiparticle pair is close to that of the two-particle problem for $\epsilon=2$ as seen by comparison with the two-particle result at $E=0$ also plotted in Fig. 2.

\footnotetext{
* Note that the single-particle localization energy should be evaluated at half the pair energy. We generated the singleparticle localization lengths for these energies numerically by the recursive Green function method [10] using systems with $10^{8}$ sites.

${ }^{\dagger}$ Note that we find $\xi_{2}<\xi_{1} / 2$ for $\epsilon=0$. This is a consequence of neglecting the first factor in Eq. (5) 8). However, there is indeed no delocalization effect in this case, because the range of both factors in (E) is independent of $u$ and $W$.
}

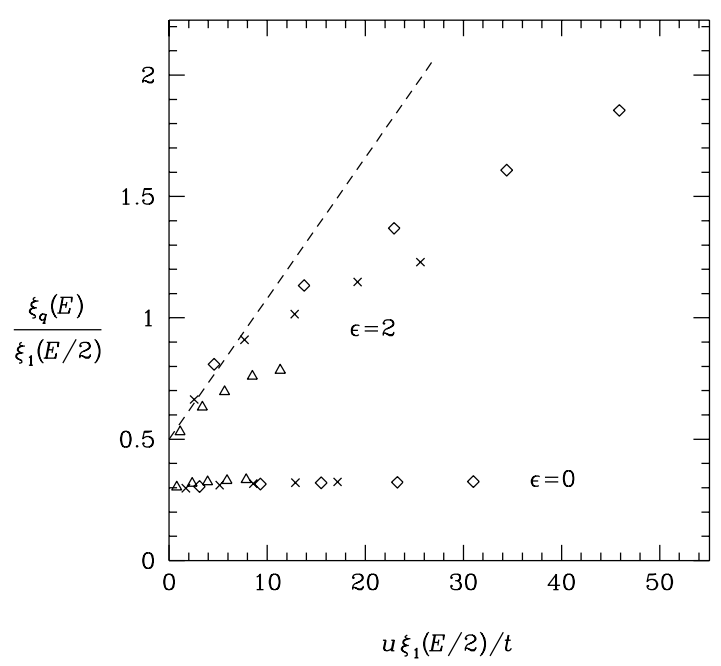

FIG. 2. Plot of $\xi_{q}(E) / \xi_{1}(E / 2)$ vs $u \xi_{1}(E / 2) / t$ for two different excitation energies. Five values of $u$ are included for each of the three values of disorder $W=3$ (triangles), $W=2$ (crosses), and $W=1.5$ (diamonds). The two-fermion effect is also shown for comparison (dashed line). Evidently, the delocalization effect has disappeared at the Fermi energy and is recovered with increasing excitation energy. One also observes deviations from the scaling behavior of the two-fermion case.

In view of the absence of the delocalization effect at zero-excitation energy, it is important to identify the relevant scale on which the effect is recovered with increasing excitation energy. To investigate this question, we have considered the enhancement of $\xi_{q}$ relative to the enhancement of $\xi_{2}$ for the two-particle problem as measured by

$$
R_{u, W}(\epsilon)=\frac{\xi_{q}(u)-\xi_{q}(u=0.1)}{\xi_{2}(u)-\xi_{2}(u=0.1)} .
$$

We have computed this quantity as a function of the excitation energy $\epsilon$ for different disorder and interaction strengths. Between $\epsilon=0$ and $\epsilon=2$, the quantity should vary roughly between zero and one. Figs. $3(\mathrm{a})$ and (b) show that $R_{u, W}(\epsilon)$ at fixed $u$ is independent of the disorder strength $W$. Moreover, a comparison of Figs. 3(a) and (b) indicates that $R_{u, W}(\epsilon)$ is also independent of the interaction strength $u$. This implies that the bandwidth $t$ is the relevant scale on which the delocalization effect is recovered with increasing excitation energy. This result is in qualitative agreement with the prediction from Thouless-type arguments by Imry [6].

Our results were obtained using a simple approximation to the two-particle Green function. Even within im-

\footnotetext{
${ }^{\ddagger}$ The accuracy of the data for $R_{u, W}(\epsilon)$ is limited because both numerator and denominator in (6) are small numbers.
} 


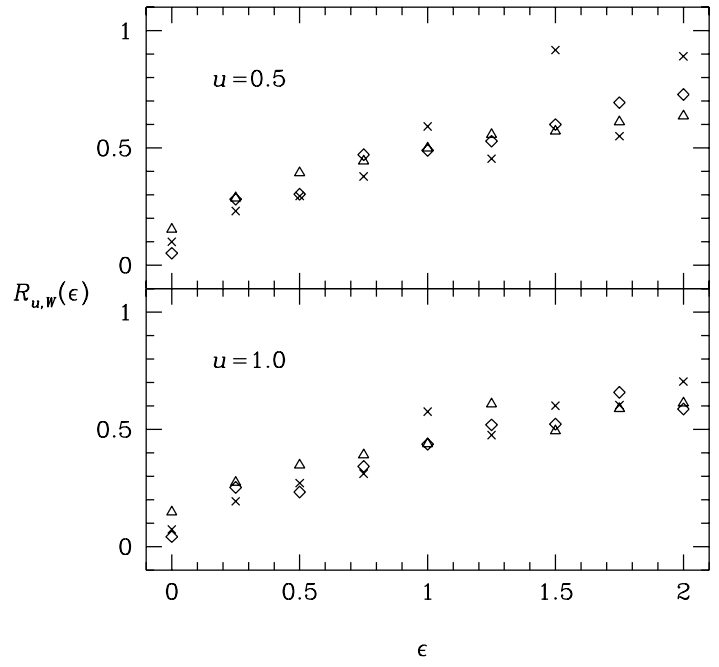

FIG. 3. Plot of the delocalization effect $R_{u, W}(\epsilon)$ as defined in Eq. (6) as a function of excitation energy for two values of $u$ and three values of disorder $W=3$ (triangles), $W=2$ (crosses), and $W=1.5$ (diamonds). Note that $R_{u, W}(\epsilon)$ is insensitive to both disorder and interaction strength.

proved approximations we do not expect a delocalization effect analogous to that for two particles to reappear at low excitation energies because the suppression of the two-particle effect at finite density is entirely due to the Pauli principle. This expectation is also in accord with standard phase-space arguments for the degenerate Fermi system. On the other hand, our approximation does not allow us to exclude the possibility that the localization properties of the Anderson insulator are strongly affected by true correlation effects.

We conclude by discussing the physical implications of our results. For thermodynamic and low-frequency transport properties in linear response, the relevant excitation energies are of the order of the temperature. In these cases, the maximal delocalization of quasiparticle pairs is not expected to be observable since the bandwidth will generally be much larger than the temperatures at which quantum-interference effects such as Anderson localization are observable. In this sense, the quasiparticle delocalization at finite density is significantly weaker than the two-particle effect. However, it may still be possible to observe the incipient delocalization at excitation energies which are low compared to the bandwidth. In particular, this may be possible in higher dimensions [6] where the delocalization effect is expected to be more pronounced and where the single-particle localization length can become exceedingly large (e.g., close to the Anderson transition). Finally, we note that there may also be a possibility of detecting the delocalization of quasiparticle pairs in high-frequency experiments.

We enjoyed helpful discussions with G. Barkema, J. Müller, and H.A. Weidenmüller.
[1] For a review, see P.A. Lee and T.V. Ramakrishnan, Rev. Mod. Phys. 57, 287 (1985).

[2] For a review, see D. Belitz and T.R. Kirkpatrick, Rev. Mod. Phys. 66, 261 (1994).

[3] See E.K. Riedel and F. von Oppen, Phys. Rev. B 47, 15449 (1993) and references therein.

[4] D.L. Shepelyansky, Phys. Rev. Lett. 73, 2607 (1994). See also F. Borgonovi and D.L. Shepelyansky, preprint (1995).

[5] O.N. Dorokhov, Zh. Eksp. Teor. Fiz. 98, 646 (1990) [Sov. Phys. JETP 71, 360 (1990)].

[6] Y. Imry, Europhys. Lett. 30, 405 (1995).

[7] K. Frahm, A. Müller-Groeling, J.-L. Pichard, and D. Weinmann, Europhys. Lett. 31, 169 (1995); D. Weinmann, A. Müller-Groeling, J.-L. Pichard, and K. Frahm, preprint (1995).

[8] F. von Oppen, T. Wettig, and J. Müller, preprint condmat/9504016 (1995).

[9] A.L. Fetter and J.D. Walecka, Quantum theory of many particle systems, McGraw-Hill (New York, 1971).

[10] B. Huckestein, Rev. Mod. Phys. 67, 357 (1995). 